\author{
„BISLANG WURDE FÜR DAS CHE RANKING DIE BIOCHEMIE DEN \\ FÄCHERN BIOLOGIE, CHEMIE ODER PHARMAZIE ZUGERECH- \\ NET, JE NACH UNIVERSITÄRER STRUKTUR UND FACHBEREICHS- \\ ZUSCHNITT. DURCH DIE GETRENNTE ERHEBUNG DES CHE HOCH- \\ SCHULRANKINGS KANN SICH DIE BIOCHEMIE JETZT BESSER ALS \\ EIGENES FACH DARSTELLEN UND PROFILIEREN.“
}

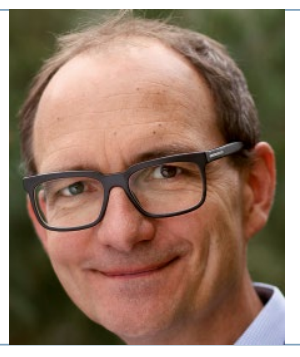

Johannes M. Herrmann

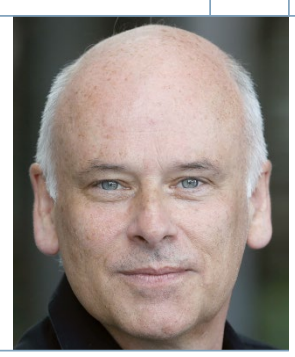

Robert Tampé

\title{
Die Biochemie-Studiengänge bekommen mehr Sichtbarkeit
}

DOI: $10.1007 / \mathrm{s} 12268-021-1559-7$

(C) Die Autoren 2021

Die Bedeutung der molekularen Lebenswissenschaften hat in den vergangenen Jahrzehnten beeindruckend zugenommen. Kaum ein anderes Gebiet der Wissenschaft ist mit einer vergleichbaren Dynamik gewachsen wie dieser Bereich im Schnittfeld von Biologie, Chemie, Physik, Medizin und Biotechnologie. Die große generelle Relevanz der molekularbiologischen Forschung wird uns in den Erfolgsmeldungen und Rückschlägen bezüglich der Bekämpfung der Corona-Krise momentan eindrücklich vor Augen geführt, wenn Nachrichtensprecher und Politiker allabendlich von PCR-Tests, Antikörpertitern und Mutationen des Spike-Proteins sprechen.

Durch diese Attraktivität und Bedeutung erleben der Studiengang Biochemie und seine verwandten Disziplinen Molekulare Medizin, Molecular Life Sciences, biomedizinische Chemie oder Chemische Biologie einen enormen Zuwachs an Studierenden. Der erste eigenständige Studiengang für Biochemie in Deutschland wurde 1962 in Tübingen gegründet und war über viele Jahre nur von einer Handvoll Studierender pro Jahrgang belegt. Heute werden diese Biochemie-Studiengänge an über 30 Studienorten in Deutschland als grundständige Bachelor- oder als aufbauende Master-Programme angeboten. Viele davon sind Numerus-clausus(NC)-Studiengänge mit Auswahlgesprächen und hohen Absolventenzahlen - und die Tendenz ist stetig steigend.

Um dieser Entwicklung Rechnung zu tragen, hat das Centrum für Hochschulentwicklung CHE entschieden, die für das CHE Ranking 2021 der Fächer Biologie und Chemie erhobenen Daten zum ersten Mal für das Fach Biochemie getrennt auszuweisen. Die Ergebnisse dieser Evaluation werden Anfang Mai im ZEIT-Studienführer veröffentlicht und sind anschließend auch online abrufbar unter https://ranking.zeit.de/che/de.

Alle drei Jahre führt das CHE fachspezifische Befragungen durch, für die zum einen Angaben der Hochschulen zu Studienmöglichkeiten und -bedingungen erfasst werden und zum anderen Befragungen von Bachelor-Studierenden des zweiten und dritten Studienjahrs der entsprechenden Fächer durchgeführt werden. Bei der aktuellen Befragung machten dabei fast 35.000 Studierende Angaben zu Lehrangeboten, Studierbarkeit und vielen spezifischen Aspekten des Studiums. Aus den Angaben der Hochschulen und der Studierendenbefragung erfolgt dann das
CHE Ranking, das vielen Studierenden als Orientierungshilfe bei der Wahl des Studienorts dient.

Über die Details der Erhebungen des CHE Rankings gibt es immer wieder lebhafte Diskussionen. Auch mag man generell über die Sinnhaftigkeit der Reihung von Studienorten streiten. Aber diese Vergleiche helfen, Stärken und Schwächen sowie die spezifischen Profile verschiedener Studiengänge besser zu erkennen neben deren wohlklingenden neudeutschen Namen. Da hier die Erhebung vornehmlich auf den Angaben der Studierenden beruht, unterscheidet sich das CHE Ranking oft deutlich von anderen Ranglisten, die aufgrund von Reputation, von Publikationen oder von Drittmitteln zustande kommen. Für diejenigen von uns, die für die Konzeption und Weiterentwicklung von Studiengängen verantwortlich sind, kann das CHE Ranking wichtige Fingerzeige geben.

Bislang wurde für das CHE Ranking die Biochemie den Fächern Biologie, Chemie oder Pharmazie zugerechnet, je nach universitärer Struktur und Fachbereichszuschnitt. Durch die getrennte Erhebung des CHE Rankings kann sich die Biochemie jetzt besser als eigenes Fach darstellen und profilieren. Für die Sichtbarkeit der Biochemie und verwandter Studiengänge als eigenständiges und wichtiges Fach ist daher die aktuelle getrennte Erhebung des CHE ein großer Erfolg.

Wir waren im vergangenen Sommer von Frau Dr. Berghoff, der Leiterin der nationalen Rankings beim CHE, eingeladen die Erhebung der Biochemie von der fachlichen Seite zu begleiten. Ein erster Überblick über die Ergebnisse wurde uns bereits präsentiert. Dabei war bemerkenswert, dass an allen teilnehmenden Hochschulen die Studierenden mit den Studienbedingungen zufrieden oder sehr zufrieden waren. Insgesamt wurde also das Biochemiestudium als ein attraktives Studium eingeschätzt.

Die jetzt erscheinende Erhebung ist allerdings nur eine Pilotstudie, deren Daten aus den Befragungen der Biologie und Chemie extrahiert wurden. Aussagekräftigere Ergebnisse sind zu erwarten, wenn in drei Jahren eine vollständige Erhebung für die Biochemie erfolgt. Wir hoffen, dass daran möglichst viele Studienorte teilnehmen und sind schon jetzt auf das Ergebnis gespannt.

Angesichts der Wissensexplosion in den Lebens- und Naturwissenschaften ist einerseits eine weitere Spezialisierung und Aufsplitterung in verschiedene Fächer unvermeidlich. Anderer- seits erfordert dies eine immer enger werdende Interaktion und Kooperation der verschiedenen naturwissenschaftlichen Fächer, um den notwendigen wissenschaftlichen Austausch zu fördern und Studierenden ein breites, umfassendes Bildungsfundament mit einer zunehmenden Internationalisierung zu bieten. Dies zu gestalten und umzusetzen bleibt eine Herausforderung und spannende Aufgabe.

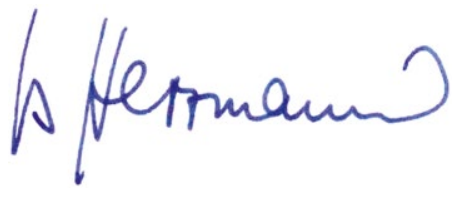

Johannes M. Herrmann, Zellbiologie, TU Kaiserslautern

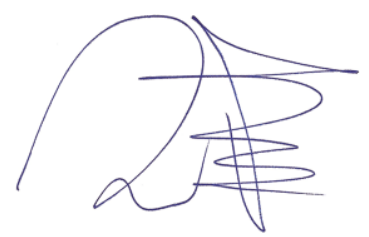

Robert Tampé, Zelluläre Biochemie, Goethe-Universität Frankfurt

Funding note: Open Access funding enabled and organized by Projekt DEAL. Open Access: Dieser Artikel wird unter der Creative Commons Namensnen-
nung 4.0 International Lizenz veröffentlicht, welche die Nutzung, Vervielfältigung, Bearbeitung, Verbreitung und Wiedergabe in jeglichem Medium und Format erlaubt, sofern Sie den/die ursprünglichen Autor(en) und die Quelle Format erlaubt, sofern Sie den/die ursprünglichen Autor(en) und die Quelle
ordnungsgemäß nnennen, einen Link zur Creative Commons Lizenz beifügen und angeben, ob Änderungen vorgenommen wurden. Die in diesem Artikel enthaltenen Bilder und sonstiges Drittmaterial unterliegen ebenfalls der genannten Creative Commons Lizenz, sofern sich aus der Abbildungslegende nichts anderes ergibt. Sofern das betreffende Material nicht unter der genannten Creative Commons Lizenz steht und die betreffende Handlung nicht nach gesetzlichen
Vorschriften erlaubt ist, ist für die oben aufgeführten Weiterverwendungen des Materials die Einwilligung des jeweiligen Rechteinhabers einzuholen Weitere Details zur Lizenz entnehmen Sie bitte der Lizenzinformation auf http://creativecommons.org/licenses/by/4.0/deed.de.

Korrespondenzadressen:

Prof. Dr. Johannes M. Herrmann

TU Kaiserslautern

Erwin-Schrödinger-Straße 13

D-67663 Kaiserslautern

hannes.herrmann@bio.uni-kl.de

www.bio.uni-kl.de/zellbiologie

Prof. Dr. Robert Tampé

Goethe-Universität Frankfurt

Max-von-Laue-Straße 9

D-60438 Frankfurt a. M.

tampe@em.uni-frankfurt.de

www.biochem.uni-frankfurt.de 